\title{
The Multidisciplinary Approach to Fragility Fractures Around the World: An Overview
}

\author{
David Marsh, Paul Mitchell, Paolo Falaschi, \\ Lauren Beaupre, Jay Magaziner, Hannah Seymour, \\ and Matthew Costa
}

\subsection{Introduction}

The opening chapter of the first edition of this book [1] told the story of the early evolution of orthogeriatric co-management in the UK and the beginnings of its spread around the world. It covered evidence accumulated up to 2016. That history will not be repeated here. Instead, this chapter aims to provide a guide to the book as a whole, which — as well as reviewing the more recent evidence-will consider the basic competencies that an effective orthogeriatric approach should deliver and propose how they might be supplied in health economies with fewer resources, in particular fewer geriatricians.

We are considering the orthogeriatric approach in its wider sense, covering the entire post-fracture pathway, including rehabilitation and secondary prevention, as well as multidisciplinary co-management of the acute fracture episode.

Already in 2015, a significant number of published studies showed better outcomes and improved cost-effectiveness with orthogeriatric co-management [2]. As Fig. 1.1 shows, the accumulation of further evidence since then has accelerated and there have now been almost 3500 publications in the decade beginning 2010, albeit not all uniformly positive in their assessment of the approach.

This chapter is a component of Part 1: Background.

An explanation of the grouping of chapters in this book is given in this chapter.

D. Marsh ( $\square)$ P. Mitchell · P. Falaschi - L. Beaupre · J. Magaziner · H. Seymour · M. Costa Fragility Fracture Network, Zurich, Switzerland e-mail: d.marsh@ucl.ac.uk; paul.mitchell@ synthesismedical.com; paolo.falaschi@fondazione.uniroma1.it; lauren.beaupre@albertahealthservices.ca; jmagazin@ epi.umaryland.edu; hannah.seymour@health.wa.gov.au; matthew.costa@ndorms.ox.ac.uk 


\section{Cumulative publications}

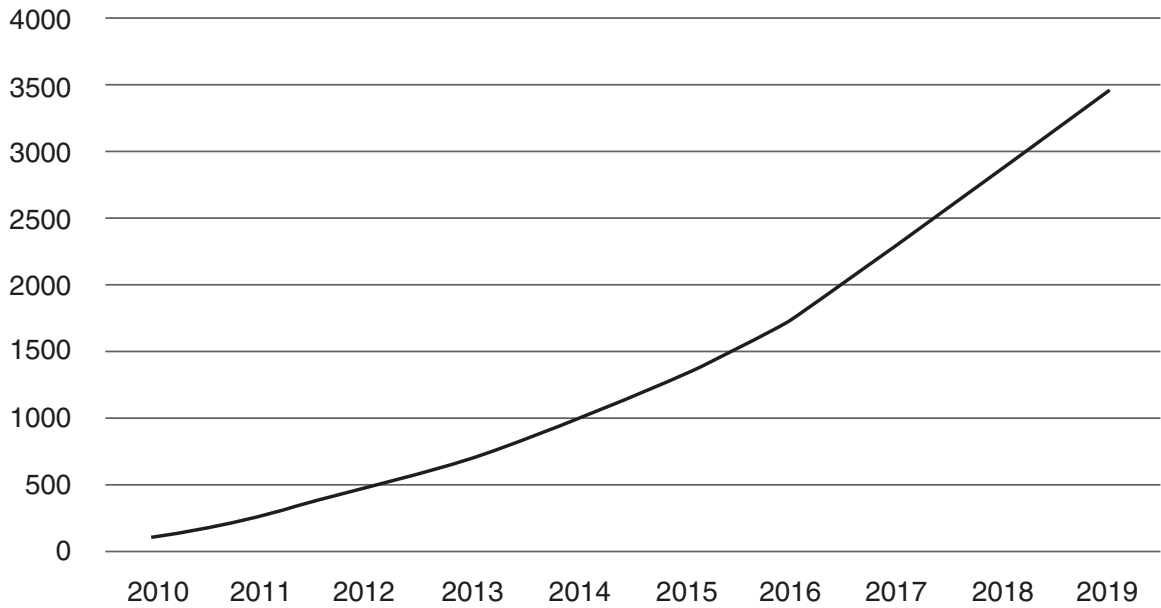

Fig. 1.1 Cumulative numbers of citations for keyword 'orthogeriatrics' in Google Scholar

One important publication in 2017 was the UN report on World Population Prospects [3]. Among other things, this gives-for each country-the Old-Age Dependency Ratio: The number of people aged 65 years or older per 100 persons of working age (15-64). As Fig. 1.2 shows (giving Thailand as a somewhat extreme example), nothing illustrates more dramatically the suddenness of the demographic change the world will experience.

The curves are a little less steep in the developed healthcare economies because the ageing of the population has already been progressing in those territories for longer. In Africa, the ratio does not rise so high in the current century but otherwise, in most regions, levels of 40-60 are expected by 2100 . Thus - at this moment in history-humankind is en route to a new demographic era.

The challenge of the ageing population, in terms of predicted population incidence of fragility fractures, is described in Chap. 2. However, the Old-Age Dependency Ratio adds a further dimension, stressing the societal consequences of ageing. The implications include:

- Society will need older people to be independent for much longer in the future. Prevention of fragility fractures and restoration of function after those fractures that do occur can make a significant contribution to that independence

- Mere survival after fracture is not going to be enough; we have to provide much more effective rehabilitation, so that 'dependency' is reduced/postponed: quality of life being more important to patients than longevity per se

- The change, and thus the pressure on health and social care services, is going to be extremely rapid; we have to start adapting and adopting measures immediately 


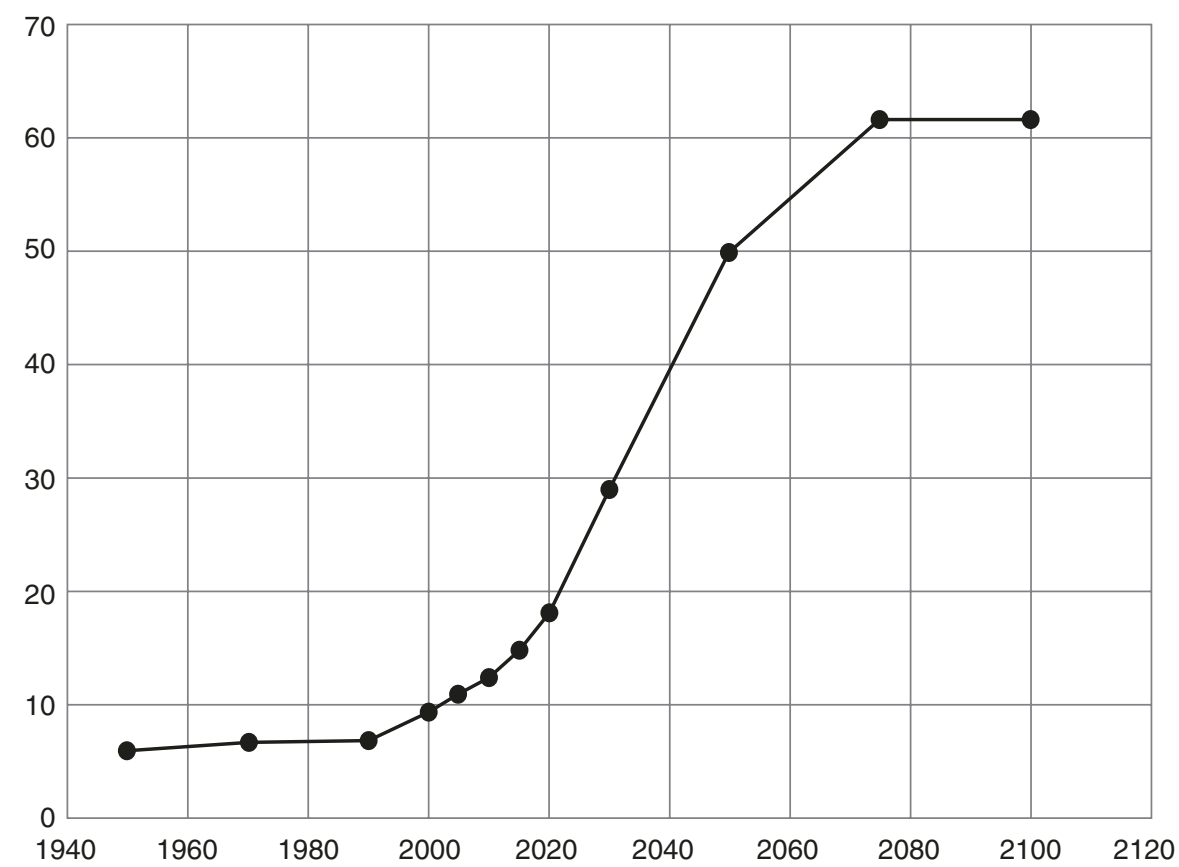

Fig. 1.2 The previous and projected Old-Age Dependency Ratio for Thailand (see text). (Data from [3], with permission)

\subsection{General Developments Since 2016}

The explosion of data generation indicates that clinical leaders all over the world are recognising the challenge and rising to it. However, it was also accompanied by other positive developments.

\subsubsection{The Global Call to Action}

At the conclusion of the 6th Global Congress of the Fragility Fracture Network (FFN) in Rome in 2016, five other organisations ${ }^{1}$ joined with FFN in laying out their vision for the following decade of work in fragility fractures. This led to the collaborative writing of a Global Call to Action, which was published in 2018

\footnotetext{
${ }^{1}$ The European Federation of National Associations of Orthopaedics and Traumatology (EFORT), European Union Geriatric Medicine Society (EuGMS), International Collaboration of Orthopaedic Nursing (ICON), International Geriatric Fracture Society (IGFS) and the International Osteoporosis Foundation (IOF).
} 
[4] - after it had been endorsed by 81 relevant professional associations, either international or from the countries with the largest populations (Brazil, China, India, Japan and USA). The recommendations in this paper can be summarised as four 'pillars':

1. Multidisciplinary care of the acute fracture episode along orthogeriatric lines

2. Excellent rehabilitation to recover function, independence and quality of life, starting immediately but continued long term

3. Reliable secondary prevention after every fragility fracture, addressing falls risk as well as bone health

4. Formation of multidisciplinary national alliances to promote policy change that enables the above three

Following publication, invitations to relevant national professional associations to endorse the Call to Action have been made; the total extent of global endorsement can be followed on the FFN website (www.fragilityfracturenetwork.org).

This succinct description of the entire sequence that should be triggered by a fragility fracture, plus the fourth-enabling — pillar, has effectively expanded the meaning of 'the orthogeriatric approach' to encompass the total response, not just the first phase. This integrated concept is well described by Pioli et al. [5], arguing that all elements are required in order to optimise patients' recovery from their fracture.

\subsubsection{The Formation of National FFNs}

The fourth pillar, multidisciplinary national alliances, took some time to achieve in the UK and other pioneer countries. Now, clinical leaders in countries wishing to take this path can speed up the process considerably by creating National FFNs. The National FFN is not the multidisciplinary national alliance per se-it is the catalyst to achieve it; the alliance has to be between the mainstream national professional associations, which the National FFN must never try to replace. The Memorandum of Understanding between the British Orthopaedic and Geriatrics organisations-described in the first edition [1] - is an early example of this approach.

The creation of National FFNs is considered the most effective method for ensuring that the Call to Action is actually implemented, rather than gathering dust on a shelf. The FFN is, therefore, promoting their formation, through a series of Regional Expert Meetings, where leaders from neighbouring countries can share experience, strategies for clinical and policy implementation, and encouragement. This approach constitutes the FFN's Regionalisation Strategy and its current status can also be found on the FFN website (www.fragilityfracturenetwork.org). A Guide to Formation of National FFNs is available at https://www.fragilityfracturenetwork. org/regionalisation/. 


\subsubsection{Initiation of New Hip Fracture Registries}

A number of countries have adopted the approach pioneered in Sweden and then the UK, where a consensus agreement on quality standards for hip fracture care was accompanied by the creation of a patient-level audit tool for measurement of compliance with those standards. There is increasing evidence (reviewed in Chap. 19) that good quality audit data linked to quality indicators reduces mortality and improves the quality of life.

However, ensuring the completeness and quality of the data requires dedication and appropriate resources. Ideally, this involves the employment of clinicallytrained coordinators who can advise data-entry personnel in busy fracture units. Such advisors are also ideally placed to promote the philosophy behind the quality standards and the logic of orthogeriatric co-management as the best way to deliver them. In the absence of clinical advisors, a critical analysis of the quality of data that is accumulated is needed, along with an assessment of the potential harm done by relying on data that is subsequently demonstrated to be unreliable. Certainly, the idea that the installation of a registry/audit system is some kind of magic wand-that will automatically raise the quality of care-needs to be resisted.

\subsubsection{Implications of These General Developments for the Design of This Second Edition}

At a meeting held in Oxford, following the 2019 Global Congress, the authors of the chapters in this book got together with other fragility fracture activists to consider how the second edition needed to be modified to properly take into account these developments. The main conclusions were:

- The chapters should be grouped according to the three clinical pillars of the Call to Action described earlier, with comprehensive cross-referencing between chapters to avoid too much repetition. The fourth pillar will be covered in other publications from the FFN

- There should be a background section covering epidemiology, osteoporosis, frailty and sarcopenia

- There should be a cross-cutting section for the role of nurses, audit and nutrition, which are relevant in all three phases

- For each of the pillars, there should be:

- up-to-date evaluation of the current evidence for the best practice where resources are available

- an analysis of what is really fundamental and critical to achieve in each pillar, that can be put in place even where resources are scarce

- advice on how to progress from a minimalist implementation to a more extensive one, as experience accumulates and resources become available 
- Although the book necessarily has to emphasise the principles within each pillar, allowing readers to shape the implementation in their healthcare system, where possible a sequence of essential practical steps towards implementation should be recommended

\subsection{Background: Chaps. 2-4}

\subsubsection{Epidemiology of Fractures and Social Costs: Chap. 2}

In addition to the re-shaping of society in terms of the Old-Age Dependency Ratio described earlier, the ageing population also has a direct effect on the population incidence of fragility fractures. This is particularly true of hip fractures, for which increasing age is a powerful risk fracture, independent of bone mineral density, and which tend to occur at later ages. Even in countries where the age-specific incidence of hip fractures is static or falling, the ageing population completely overwhelms that, so that population incidence is climbing everywhere. Chapter 2 explores that issue across the world and describes the very heavy costs of the condition, for patients, their carers and the health services.

The remaining two background issues are the underlying drivers of fragility fractures that stem from ageing: osteoporosis, which causes bone fragility (Chap. 3) and frailty, linked to sarcopenia (Chap. 4).

\subsubsection{Osteoporosis in Older Patients: Chap. 3}

Chapter 3 describes the basic biology of bone and how and why it deteriorates in osteoporosis. However, this does not lead to symptoms per se, until there is a fracture. The risk factors for fracture are manifold, in addition to the loss of bone mineral density, but there is a consistent increase in fracture risk as the T-score diminishes. Age plays a powerful role-independently of BMD, as do a history of previous fracture and a family history of fracture. The various modalities for estimating bone quality and fracture risk are discussed in this chapter (but also in Chap. 14). Similarly, the principles of treatment aimed at reducing fracture risk are covered in Chap. 3, but also in more detail in Chap. 15.

\subsubsection{Frailty and Sarcopenia: Chap. 4}

Frailty is a syndrome that affects the whole of an older person's physiology. As described below (Sect. 1.4), the existence of frailty in many patients presenting with fragility fractures is the prime reason for the need of orthogeriatric co-management, since the discipline of geriatric medicine specialises in it. This chapter, written by two leading European experts, defines its nature, its epidemiology and aetiology and its implications for clinical care. The prominent role of sarcopenia in frailty (analogous to that of osteoporosis in fragility) is explained. 
The practical implications of frailty and sarcopenia for falls, fractures and recovery after fractures are explored, including the planning of rehabilitation and discharge from the hospital.

There are close links epidemiologically, biologically and clinically between frailty, sarcopenia, poor bone health and the geriatric syndrome of falls. Older people who have had a fall and/or a fracture should be assessed for frailty and sarcopenia to better develop a care plan. This calls for an integrated clinical approach to the prevention and treatment of fragility fractures.

\subsection{Pillar I: Co-Management in the Acute Episode- Chaps. 5-11}

The basic point about patients presenting with fragility fractures, especially older patients with hip fractures, is that most of them are suffering from two separate issues. The first is the fragility of their bone, due to osteoporosis or osteopenia, which has allowed the fracture to occur with minimal trauma. The second is frailtyof their whole body, which as Chap. 4 explains, weakens their capacity to respond to stress and is associated with comorbidities. It is unfortunate that several languages use the same word to describe these two entities, since they are totally different; the first being a biomechanical issue, the second physiological.

Orthopaedic surgeons are trained to deal with the fragility; geriatricians are trained to address frailty (other medical disciplines can also learn to do so). The older patients with fragility fractures, therefore, need the application of both skillsets if they are to emerge from the experience with good health and function. That is the basic argument for orthogeriatric co-management. It is expanded by the need to include other disciplines in a multidisciplinary team, particularly anaesthetists, nurses and physiotherapists.

The operative and non-operative treatment of incident fractures is clearly an enormous subject by itself, and one that is more appropriately dealt with in other publications. In this book, we have chosen two examples that illustrate important principles. The first is hip fracture, which we have chosen because (i) it is the index fracture for fragility fractures generally in much of the epidemiological and health economic literature, (ii) it accounts for the majority of in-patient costs of fragility fractures generally and (iii) it was historically, and remains, the prime setting for orthogeriatric co-management. The second is a proximal humeral fracture, which illustrates the paucity of solid evidence, in many fracture types, that operative fixation, with the maximal restoration of skeletal anatomy, leads to a better clinical result.

\subsubsection{Establishing an Orthogeriatric Service: Chap. 5}

Chapter 5 tackles the challenge of putting such a multidisciplinary service into practice, using a well-attested change-management methodology. It is as much a political as a medical challenge since it inescapably involves sharing between disciplines 
the care of patients traditionally under the sole care of one discipline-orthopaedic surgery. Diplomacy is required. This chapter describes an approach, based on eight steps:

1. Process mapping the hip fracture pathway

2. Identify a core multidisciplinary team and form a steering group

3. Analyse and review the patient pathway

4. Evaluate the resources required to drive change within the organisation

5. Develop the business case for the orthogeriatric service

6. Implementing and sustaining the service

7. Collect evidence of service improvement-Audit

8. Embrace the support of regional, national and international organisations

The sequence of chapters after that follows the patient journey in the acute episode: pre-hospital—pre-operative_-anaesthetic_-surgery-post-operative.

\subsubsection{Pre-hospital Care and the Emergency Department: Chap. 6}

Chapter 6 covers the period between the patient's injurious fall and their admission to the hospital. It is consistent with the well-developed methodology that is applied to high energy trauma victims and other emergencies, with prioritised primary and secondary surveys. There is an emphasis on rapid transfer, but with due regard to minimising pain through gentle immobilisation of the limb and gentle driving of the ambulance. History-taking at the scene and on the journey is a valuable contribution to the holistic picture of the patient and their circumstances that will guide future management and discharge. Pain relief is paramount but must not be gained at the expense of opiate overdosing. Paramedic-administered pre-hospital fascia iliaca compartment blockade can be seen on the horizon but, for the most part, that is currently something to be done once the Emergency Department has been reached.

The situation in developed countries, with short journeys in modern vehicles with highly-trained crews, is radically different from that in many low-resource settings in developing countries. There, it may be a difficult and prolonged journey to get the patient to the hospital, but the principles and goals of safety, painrelief and adequate fluid management are universal. So the challenge is to implement effective protocols that deliver those goals as completely as possible in each setting. In some areas, every effort is made to minimise the time spent in $\mathrm{A} \& \mathrm{E}$ as this is a busy and often chaotic area where an immobile patient with frailty may come to harm with delirium and pressure damage. However, appropriate triage with recognition and management of acute inter-current conditions must not be missed. In contrast, in some countries, A\&E physicians may take the lead in pre-operative optimisation. 


\subsubsection{Perioperative Orthogeriatric Care: Chaps. 7 and 11}

The substantial meat of orthogeriatric wisdom and experience is to be found in Chaps. 7 and 11, covering respectively pre- and post-operative medical management. These chapters have been written by authors working in developed countries where there has been a drive to identify and proactively manage patients with frailty with the development of the specialty of geriatric medicine. Orthogeriatrics has evolved as a sub-specialty, with orthogeriatricians working closely with orthopaedic surgeons and anaesthetists, in addition to the multidisciplinary team.

These chapters are written aiming to describe gold standard management. It is important to acknowledge that many countries, and particularly those with the most significant challenges, where an epidemic of patients with hip fracture is predicted, may not have geriatric medicine as a defined specialty, and solutions to orthogeriatric care need to be imaginatively devised. This requires that the role of the orthogeriatrician be analysed and understood so that every country can find a way to ensure that patients with hip fracture and other fragility fractures, particularly in those with significant frailty, are managed appropriately.

Orthogeriatricians have often led the development of local pathways and protocols to standardise and improve care and to ensure communication between all specialists involved. This leadership role may be taken on by any member of the team but requires a real understanding of frailty and all the roles in the multidisciplinary team.

However, we must face up to the fact that there is no way that sufficient numbers of card-carrying geriatricians can be generated in time to deal with the epidemic of hip fractures that is on its way. Orthogeriatric competencies, based on recognition and understanding of frailty, must be inculcated in other species of health workers. There needs to be a wide army of 'Frailty Practitioners' who may, in different settings, be derived from other types of physicians or other professions such as nursing/pharmacy/physiotherapy/occupational therapy, albeit led and taught by geriatricians wherever possible. This is going to require a substantial shift in culture in many countries, where the empowerment of nurses and other healthcare professionals is an anathema. But the alternative, when the epidemiological predictions become reality, is chaos and misery.

Some key areas of orthogeriatric competency that are transferable from the discipline of geriatric medicine are:

- Skills in pre-operative assessment and optimisation of comorbidities: There is now a reasonable evidence base, as covered in Chaps. 7 and 11, that can be protocol-driven and delivered by those with basic training (junior doctors, advanced nurse practitioners) liaising with anaesthetists

- Recognition of severe frailty with limited reversibility: About a quarter of patients presenting with hip fracture are in their last year of life. Patients with significant frailty and limited physiological reserve benefit most from an early 
operation and early mobilisation, aiming to reduce their high risk of complications. Setting realistic expectations - and appreciating when an operation is purely about managing pain rather than restoring mobility/independenceis crucial

- Continuity of care: The orthogeriatrician often oversees the patient's journey from admission through to discharge. This advocate role, requiring excellent communication with the patient, their family and all members of the team, is increasingly being undertaken by specialist nurses or others

Sandwiched between Chaps. 7 and 11, as they are in clinical reality-are the anaesthetic episode and the surgical intervention.

\subsubsection{Orthogeriatric Anaesthesia: Chap. 8}

Chapter 8 covers a lot more than the techniques of anaesthesia per se. The role of the anaesthetist is considered alongside that of the orthogeriatrician; after all, both are primarily interested in the patient's physiology. The goal is not just to bring the patient safely through the surgical procedure but also (i) to expedite readiness for surgery, (ii) to use the intraoperative, intensely-monitored phase to normalise the patient's physiology as far as possible, to maximise their ability to mobilise early and rehabilitate and (iii) to assist in ensuring good control of pain in all phases of the in-patient episode.

A simple key step, which can be a liberation in many fracture units, is to include anaesthetic colleagues in the definition of the consensus protocols governing the multidisciplinary management of fragility fractures requiring admission for surgery. The prime purpose of this is to secure the agreement of anaesthetic colleagues to a set of standardised procedures covering readiness for surgery and, as far as possible, the anaesthetic and pain management techniques to be deployed. An example of an issue where standardised techniques, established in consultation with orthogeriatric colleagues, can improve safety and early mobilisation, is the agreement to use only low doses of the local anaesthetic in spinal anaesthesia, to minimise the induced hypotension.

Anaesthetic practice also varies enormously across the world with access to trained practitioners, drugs, equipment and electricity lacking in some areas. There is a huge amount of work to do but, again, Chap. 8 summarises the evidence from developed countries and makes recommendations towards gold standard practice.

\subsubsection{Hip Fracture: The Choice of Surgery-Chap. 9}

Chapter 9 focuses on how to achieve stable fixation in the different patterns of hip fracture. As described in the first edition of this book [1], the very first paper on orthogeriatric co-management of elderly hip fracture patients, presented to the British Orthopaedic Association in 1966 was from the original orthogeriatric unit of 
Devas (a surgeon) and Irvine (a geriatrician) in Hastings, UK [6]. Already, from these first 100 cases, they asserted that a successful operation needs to make it possible for the patient to mobilise, fully weight-bearing, on the first post-operative day. The bed is a dangerous place for older patients! It is disappointing how many orthopaedic surgeons around the world, over 50 years later, still insist on prolonged bed rest for their hip fracture patients, as if maintaining the pristine beauty of the postoperative X-ray is more important than the functional recovery of the patient.

\subsubsection{Proximal Humeral Fractures: The Choice of Treatment-Chap. 10}

Many surgeons find operating on proximal humeral fractures enjoyable and satisfying. However, the recent clinical evidence suggests that their enthusiasm is not matched by improved outcomes following operative treatment. Neither operative nor conservative management leads to a great recovery of shoulder mobility and function and the relief of pain-overwhelmingly the top priority for the patientsseems to be at least as good after non-operative management. There might, however, be a place for evidence-based surgery in older patients with fracture-dislocations, articular surface fractures and fractures with no contact between the bony fragments. Evidence from clinical trials is not of the highest quality so far, with very heterogeneous cohorts, but better-designed studies are in the pipeline; perhaps 'big data' from registries may give further insights.

\subsection{Pillar II: Rehabilitation-Chaps. 12 and 13}

As pointed out in Sect. 1.1 of this chapter, one of the implications of the rapidlyincreasing Old-Age Dependency Ratio is that we need to provide much more effective rehabilitation after a fragility fracture, so that 'dependency' is reduced/ postponed. A complementary dimension is that, to the extent that post-fracture patients do become dependent, their care is going to devolve increasingly to family caregivers, since the volume of cases will overwhelm existing services in all countries.

\subsubsection{Rehabilitation Following Hip Fracture: Chap. 12}

As noted above, in Sect. 1.4.5, the most immediate goal after hip fracture surgery should be early—next-day—mobilisation. It will take time to convince surgeons in some countries that this is so. However, that is just the beginning; there needs to be a planned, individualised rehabilitation programme that starts in the hospital and carries on for an extended period-basically for the rest of the patient's life-after discharge. Ideally, this should be delivered by a multidisciplinary team, integrating physical therapy with social support, nutrition advice and so on. It is important that 
the team meets to review progress and gives the patient a sense of coordinationand optimism. Chapter 12 is an exhaustive review of the current evidence about what rehabilitative measures are effective.

Recognising that large rehabilitation teams will not be available in many lowand middle-income countries, implementation strategies for those settings, including input from families, are suggested.

The point is made that cognitively-impaired patients are among those who benefit most from robust programmes and must not be excluded from rehabilitation efforts.

\subsubsection{The Psychological Health of Patients and Their Caregivers: Chap. 13}

The physical and emotional burden of looking after an elderly relative with some degree of disablement is considerable. As might intuitively be expected, there is now good evidence that the physical and psychological health of post-fracture patients and their caregivers are interdependent. Chapter 13 addresses the issue of how their status can be assessed and how the orthogeriatric team can help.

\subsection{Pillar III: Secondary Prevention-Chaps. 14-16}

As most readers of this book will know: (i) having a fragility fracture increases the risk of another one- 'fracture begets fracture'; (ii) half of all hip fractures have been preceded by a previous fragility fracture and (iii) the risk of recurrent fracture is greatest in the first year the 'imminent refracture'. The obvious consequence of these facts is that the response to a patient presenting with any fragility fracture is not complete until there has been a determined attempt to prevent another one. This must address both osteoporosis and falls risk and it must happen as quickly as possible after the incident fragility fracture.

If this is done reliably, given that current treatments reduce fracture risk by around $50 \%$, there is the potential to prevent $25 \%$ of subsequent hip fractures; as treatments improve, this should increase. The fact that it is not done reliably-with a 'treatment gap' in the order of $80 \%$ - means that the burning issue right now is the need for reconfiguration of fracture services to capture every fragility fracture and link them to the appropriate bone health and falls prevention services.

\subsubsection{Fracture Liaison Services: Chap. 14}

The basic model for achieving this is very clear and well-documented-it is the Fracture Liaison Service. Chapter 14 focuses on how such a service should assess the magnitude of the risk of another fracture and initiate action to reduce that risk. 
It explains how such a service can be created, whatever the level of resources in any given location. Like orthogeriatric co-management generally, it is more a matter of changing attitudes than spending money. This chapter is written by leading drivers of the Capture the Fracture ${ }^{\circledR}$ campaign of the International Osteoporosis Foundation.

\subsubsection{Current and Emerging Treatment of Osteoporosis: Chap. 15}

Chapter 15 focuses on the pharmacological treatment of osteoporosis: the indications for it and the prospects for more powerful treatments than we have previously enjoyed. Gold standards of assessment in developed economies include measurement of bone mineral density, in younger fracture patients, by DXA scan; this will not be available in many low-resource settings. The authors suggest that, in such settings, the fact that a fragility fracture has already occurred can be considered sufficient grounds for initiating anti-resorptive treatment - even in younger patients. This is controversial, but all would agree that the possibility should be considered, and the decision be made on clinical judgement.

\subsubsection{How Can We Prevent Falls?-Chap. 16}

Whatever the degree of fragility conferred on the bones by osteoporosis, the vast majority of fragility fractures are nonetheless precipitated by falls (vertebral fractures being the obvious exception). The evidence base for fracture prevention by falls risk reduction is meagre compared to that for osteoporosis treatment because there has so far not been the commercial imperative for the industry to fund large scale trials. This may change whenever a realistic anti-sarcopenia agent becomes available.

However, Chap. 16 summarises a gradually-accumulating body of evidence about how an individual's risk of falling may be classified as low, intermediate or high and what treatment programmes are effective for each category. This body of evidence is related to that exhaustively described in Chap. 12-rehabilitation after hip fracture.

There is understandably great concern about falls that occur in care settings and it is recommended that all in-patients over the age of 65 be considered and treated as high risk. Patients with cognitive impairment may require special attention in addition to the high-risk programme; in advanced dementia, recurrent falls indicate the need for palliative care.

In post-fracture cases, falls clinics and programmes need to be linked to the Fracture Liaison Service and fall risk and bone health should always be considered together. 


\subsection{Cross-Cutting Issues: Chaps. 17-19}

The nursing role, nutrition and fracture audit systems are relevant in all three clinical pillars.

\subsubsection{Nursing in the Orthogeriatric Setting: Chap. 17}

Attitudes to nurses, and what they may be capable of, vary widely across the globe. In some developed economies, they have been significantly empowered, based on models of advanced training, protocol-driven care and supervision by appropriate medical specialists. This includes, in many locations, the ability to order investigations and treatments within protocols. By contrast, there are many countries where such autonomy would be anathema. What is very clear, and needs to be asserted as frequently as possible, is that the volume of fragility fracturerelated work - already now but more so in the future-is such that it cannot realistically be delivered without enhanced nurse input. There is no prospect that there will be (i) enough geriatricians on the planet to deliver orthogeriatric surveillance of all older fracture inpatients on a daily basis or (ii) enough endocrinologists for every fragility fracture patient to be assessed for secondary prevention by a doctor.

This chapter explores the key nursing roles and interventions relating to orthogeriatric nursing care. It considers how nursing care quality, focused on nurse-specific indicators, skill, education, leadership and resources, can positively impact patient outcomes in all phases of the care journey. The central nursing role in preventing and managing complications is specifically outlined.

\subsubsection{Nutritional Care of the Older Patient with Fragility Fracture: Chap. 18}

Nutrition is a subject that can be very complex in the elderly. Chapter 18 opts for the SIMPLE approach:

\begin{tabular}{ll|} 
S & Screen for nutrition risk \\
I & Interdisciplinary assessment \\
M & Make the diagnosis (es) \\
\hline P & Plan with the patient \\
L & impLement interventions \\
E & Evaluate ongoing care requirements
\end{tabular}

The essence of this approach is that, rather than requiring a highly-specialised nutritionist, a systematic assessment on the part of all the disciplines that are interacting with the patients can achieve what is needed for the nutritional care of the older patient with a fragility fracture, regardless of setting or healthcare provider. 


\subsubsection{Fragility Fracture Audit: Chap. 19}

Fragility fracture audit is key to understanding fragility fracture management, identifying areas for its improvement, and measuring the impact of clinical initiatives and service change. A hip fracture can be considered a marker for fragility fractures, and hip fracture audits indirectly show the strengths and weaknesses of fragility fracture care overall. Notably, where effective hip fracture care exists, this has a favourable impact on the care of other fragility fractures. Early established audits, clinically led, have used clinical standards and feedback on compliance with them to improve care and outcomes. Although data collection, analysis and feedback require investment, the cost per case amounts to only a very small fraction of the cost of care per case.

Because patients with fractures requiring surgical intervention, such as hip fractures, are available for some time in the hospital, it is relatively easy to collect the data needed for a registry or audit database. Capturing the necessary data for secondary prevention is a lot harder, between the fleeting outpatient clinic visits and the variable passage from secondary to primary care settings. It is also complex to link databases in such a way that recurrent fractures are reliably captured and matched with data describing secondary prevention interventions. Nonetheless, this problem must be solved, if we are ever to have the evidence needed to persuade healthcare funders to commission reliable Fracture Liaison Services for the long term. This is likely to be one of the main developments in the following phase of fragility fracture service evolution.

\subsection{Concluding Remarks}

Demographic change over the following decades presents significant challenges in many areas of medicine. However, the strong association between age, skeletal fragility and physiological frailty mean that fragility fractures will feature very prominently in that development. Furthermore, the need to extend the period of independence in people's longer lives drives us to realise that we need to (i) prevent as many fractures as possible-in which secondary prevention represents the lowest-hanging fruit and (ii) treat those that do occur in the most cost-effective way possible, with full regard to recovery of function.

Hopefully, the chapters of this book summarise the current (2020) state of these arts adequately. But equally hopefully, they will be out of date pretty soon, as progress is made.

While the book will remain as it is until a subsequent edition, the FFN will maintain, on its website www.fragilityfracturenetwork.org, two toolkits that will complement the book and be updated as experience grows. One is a Clinical Toolkit, which is concerned with the practical implementation of Pillars I-III, as described in most of the chapters of this book. The other is a Policy Toolkit, which expands Chap. 1-particularly Sect. 1.2-in other words the fourth pillar of the healthcare policy change and the work of the National FFNs. 


\section{References}

1. Marsh D (2017) The orthogeriatric approach—progress worldwide. In: Falaschi P, Marsh D (eds) Orthogeriatrics. Springer International, Cham

2. Sabharwal S, Wilson H (2015) Orthogeriatrics in the management of frail older patients with a fragility fracture. Osteoporos Int 26:2387-2399

3. Department of Economic and Social Affairs, Population Division (2017) World Population Prospects: The 2017 Revision. Volume II: Demographic Profiles. United Nations, New York.

4. Dreinhöfer KE (2018) A global call to action to improve the care of people with fragility fractures. Injury 49:1393-1397

5. Pioli $\mathrm{G}$ et al (2018) Orthogeriatric co-management-managing frailty as well as fragility. Injury 49:1398-1402

6. Devas RE, Irvine MB (1967) The geriatric orthopaedic unit. J Bone Jt Surg 49:186-187

Open Access This book is licensed under the terms of the Creative Commons AttributionNonCommercial-NoDerivatives 4.0 International License (http://creativecommons.org/licenses/ by-nc-nd/4.0/), which permits any noncommercial use, sharing, distribution and reproduction in any medium or format, as long as you give appropriate credit to the original author(s) and the source, provide a link to the Creative Commons license and indicate if you modified the licensed material. You do not have permission under this license to share adapted material derived from this book or parts of it.

The images or other third party material in this book are included in the book's Creative Commons license, unless indicated otherwise in a credit line to the material. If material is not included in the book's Creative Commons license and your intended use is not permitted by statutory regulation or exceeds the permitted use, you will need to obtain permission directly from the copyright holder.

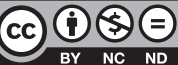

